\title{
An unusual mediastinal parathyroid carcinoma coproducing PTH and PTHrP: A case report
}

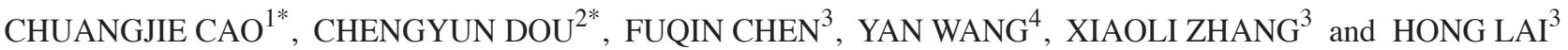 \\ ${ }^{1}$ Department of Pathology, First Affiliated Hospital of Sun Yat-sen University, Guangzhou, Guangdong 510080; \\ Departments of ${ }^{2}$ Hepatology, ${ }^{3}$ Endocrinology and ${ }^{4}$ Pathology, Qilu Hospital of Shandong University, \\ Jinan, Shandong 250012, P.R. China
}

Received March 17, 2015; Accepted April 8, 2016

DOI: $10.3892 / \mathrm{ol} .2016 .4548$

\begin{abstract}
Parathyroid carcinoma (PTCA) is a rare disease, and ectopic PTCA is particularly rare. Parathyroid hormone-related protein (PTHrP) expression in PTCA has not been previously described in the relevant literature to the best of our knowledge. The present study reports a unique case with a mediastinal parathyroid carcinoma producing parathyroid hormone (PTH) and PTHrP. A 53-year-old man presented with hyperparathyroidism symptoms, including fatigue, chest pain, dizziness, muscular soreness, polyuria, night sweats and renal stones. However, neck ultrasound revealed no significantly abnormal thyroid or parathyroid nodules. Tc99m methoxyisobutylisonitrile (Tc99m-MIBI) scintigraphy scanning indicated an ectopic mediastinal parathyroid adenoma. Histopathological examination revealed PTCA, and the tumor tissue was coproducing PTH and PTHrP. The patient underwent successful surgical operation. Serum calcium and PTH levels remained within normal ranges, and there was no tumor recurrence observed at a 3-year follow-up appointment. Although rare, ectopic parathyroid glands may lead to malignant disease. Clinical symptoms, biochemical tests, ultrasound and Tc99m-MIBI scintigraphy scanning may assist with the diagnosis of this disease. Hypersecretion of PTHrP and PTH contributed collaboratively to the pathogenesis of hypercalcemia due to PTCA. Complete surgical resection with microscopically negative margins is the recommended treatment for PTCA and offers the best chance of a cure.
\end{abstract}

Correspondence to: Professor Hong Lai, Department of Endocrinology, Qilu Hospital of Shandong University, 107 Wenhuaxi Road, Jinan, Shandong 250012, P.R. China

E-mail: laihong@medmail.com.cn

*Contributed equally

Abbreviations: PTCA, parathyroid carcinoma; Tc99m-MIBI, Tc99m methoxyisobutylisonitrile; PTA, parathyroid adenoma; AKP, alkaline phosphatase; CT, computed tomography; IHC, immunohistochemistry; $\mathrm{Ca}$, calcium

Key words: hypercalcemia, parathyroid carcinoma, parathyroid hormone-related protein, parathyroid hormone

\section{Introduction}

Parathyroid carcinoma (PTCA) is a rare malignancy, accounting for $0.005 \%$ of all cancers $(1,2)$, and ectopic PTCA is particularly rare. The incidence of ectopic parathyroid glands in healthy individuals is $\sim 6 \%$ (3), and are located in the anterior superior mediastinum (4), either within or outside the thymus and along the esophagus, into the posterior superior mediastinum (5). Therefore, ectopic PTCA is particularly rare.

Although the etiology of PTCA is unknown, history of neck radiation (6) and secondary and tertiary hyperparathyroidism caused by chronic renal failure (7) are known risk factors for PTCA. In addition, PTCA has been associated with hyper-functioning parathyroid glands (8). Of all types of parathyroid cancer, $\sim 95 \%$ are hormonally functional, and frequently exhibit profound symptoms of hyperparathyroidism with marked hypercalcemia and elevated parathyroid hormone (PTH) levels. These symtpoms include polydipsia or polyuria, myalgia or arthralgia, nephrolithiasis, weakness, fatigue, nervousness, depression, renal insufficiency, pancreatitis, peptic ulcer disease or weight loss. Bone pain, osteopenia, osteofibrosis and pathological fractures were the prominent manifestation of skeletal involvement in PTCA (9).

Parathyroid hormone-related peptide (PTHrP) causes hypercalcemia via increased calcium absorption at the kidney and increased bone resorption. PTHrP is widely expressed at low levels throughout the body in healthy individuals (10), and is also ectopically secreted at much higher levels by certain solid tumors and in several hematological malignancies (11). However, to the best of our knowledge, there are no previous reports about co-expression of PTH and PTHrP contributing to hypercalcemia in PTCA. The present study reports a unique case of a patient with an ectopic mediastinal PTCA coproducing PTH and PTHrP.

\section{Case report}

A 53-year-old man presented to Qilu Hospital of Shandong University (Jinan, China) on December 2011 with fatigue (with a 1-year history), intermittent chest pain and dizziness (with a 2-day history), and intermittent muscular soreness (with a 9-hour history). The patient also had a 2-year history of polyuria, night sweats and renal stones. There was no family 
A

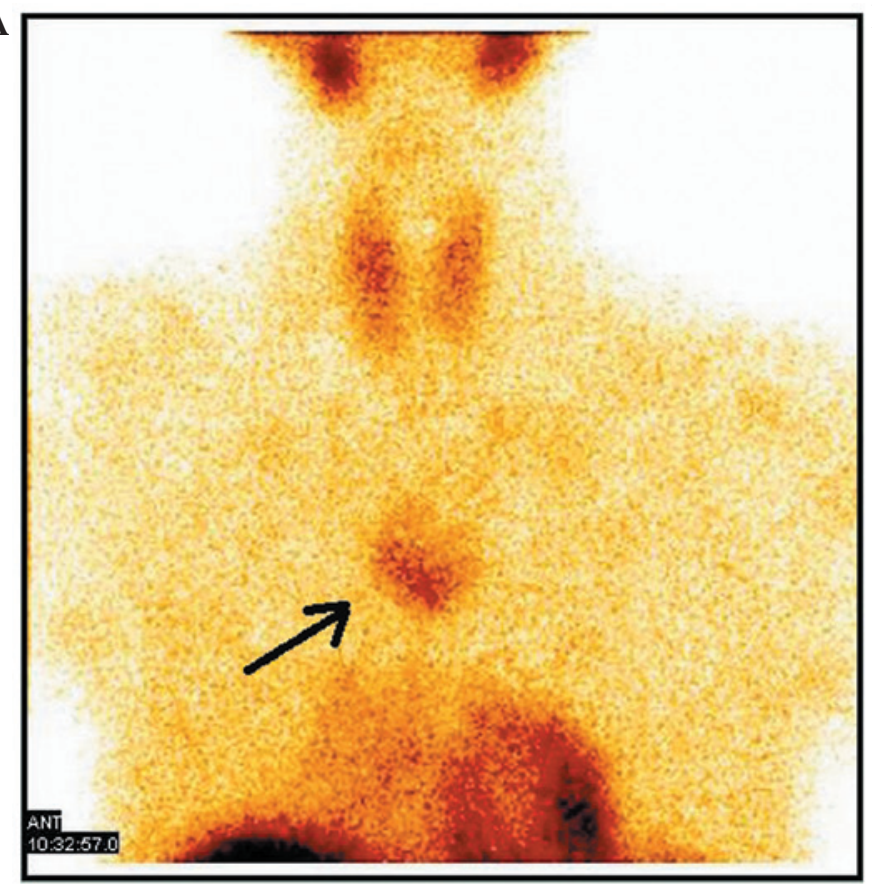

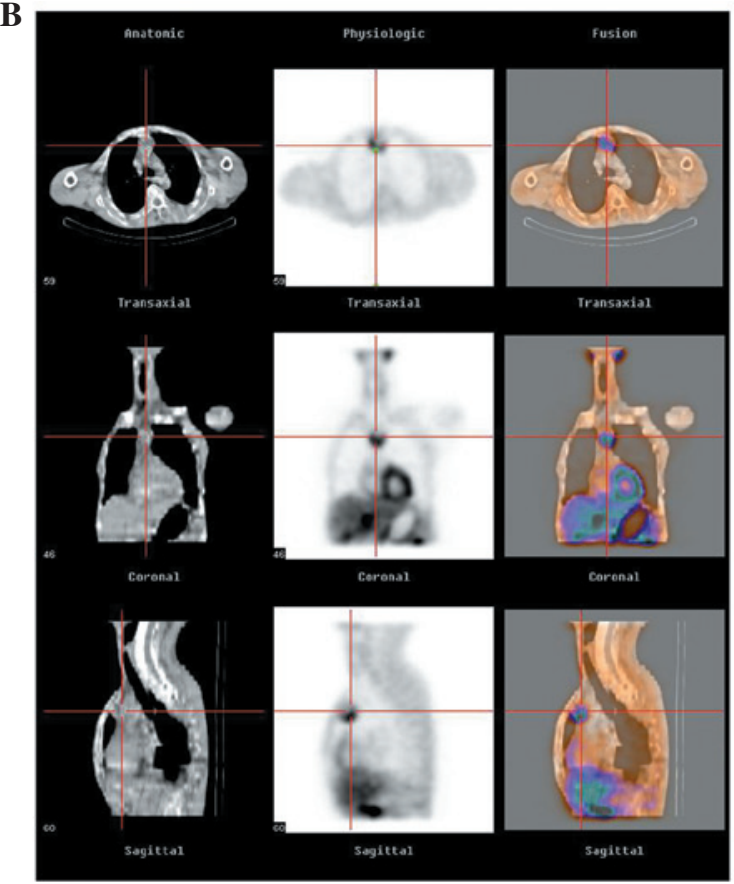

Figure 1. Tc99m-MIBI scintigraphy scan of the patient's anterior mediastinum. (A) The static state of the parathyroid carcinoma. The black arrow indicates the tumor. (B) Three-dimensional reconstruction of parathyroid carcinoma. The center of the cross represents the location of the tumor. The first column corresponds to the anatomical scanning image by computed tomography. The second column is the physiological scan by Tc99m-MIBI scintigraphy. The third column represents the fusion of the first and second columns. The first line is the transaxial tomogram, the second line is the coronal tomogram and the third line corresponds to sagittal tomography. MIBI, methoxyisobutylisonitrile.

A

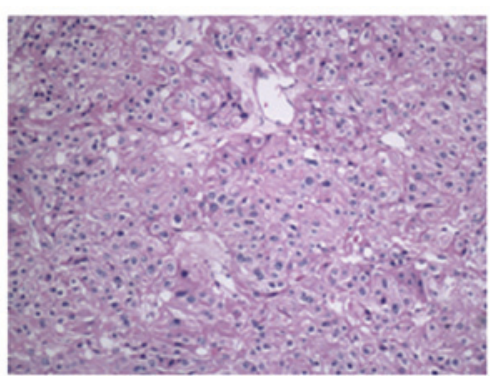

D

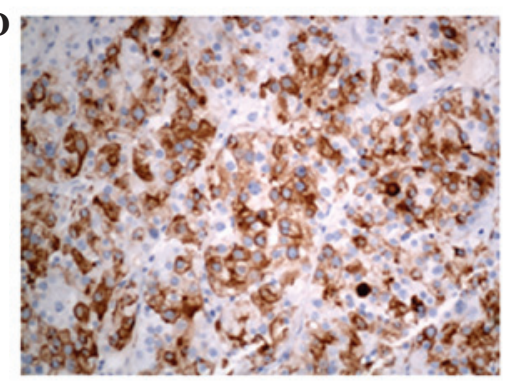

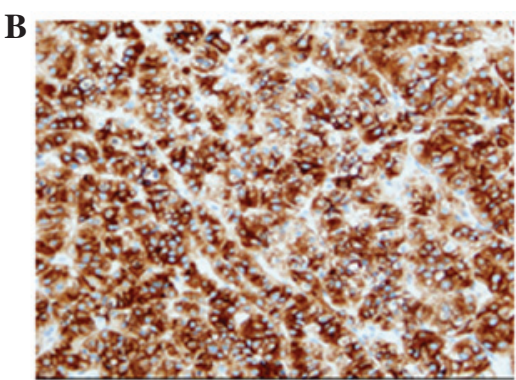

$\mathbf{E}$

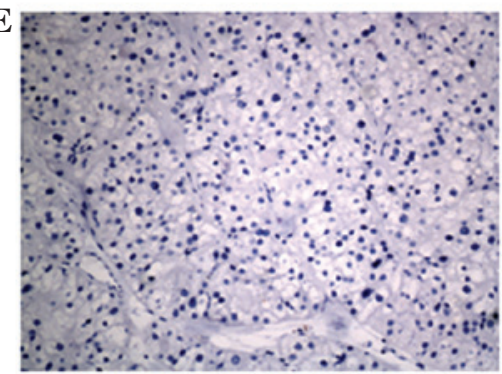

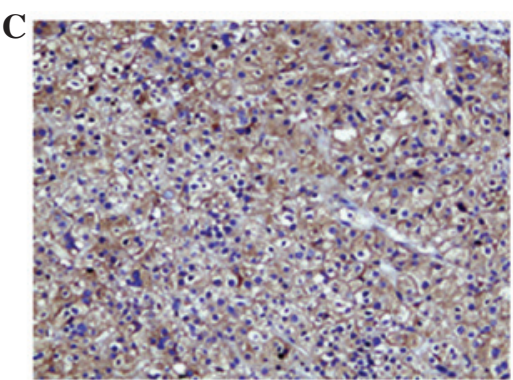

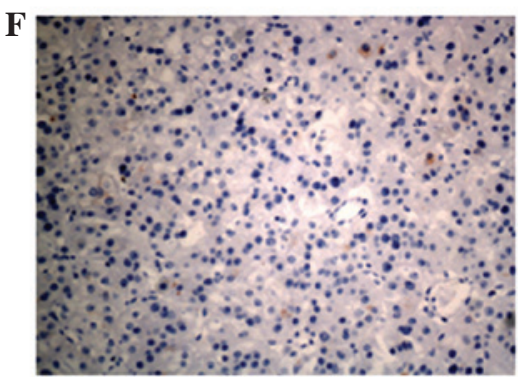

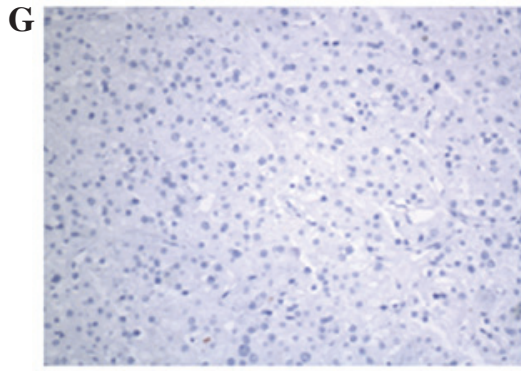
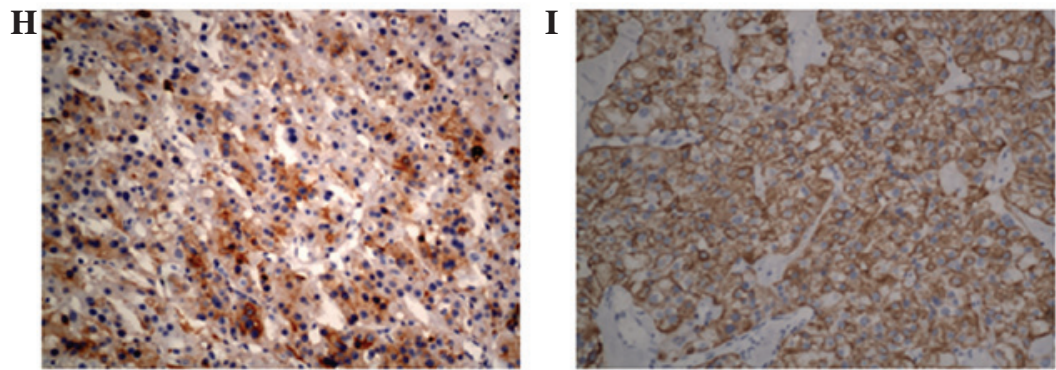

Figure 2. Histology and immunohistochemistry of parathyroid carcinoma. (A) Hematoxylin and eosin staining. (B) Positivity for parathyroid hormone. (C) Positivity for parathyroid hormone-related protein. (D) Positivity for cytokeratin. (E) Negativity for human melanoma black-45. (F) Negativity for epithelial membrane antigen. (G) Negativity for Melan-A. (H) Positivity for synaptophysin. (I) Positivity for chromogranin. Magnification, x200. 
history of renal stones or any other kidney disease. The patient had been healthy previously, with the exception of a surgery performed due to abdominal trauma caused by an accident.

A physical examination revealed an average body type (height, $175 \mathrm{~cm}$; body weight, $76.3 \mathrm{~kg}$ ) with fatigue and stable vital signs [body temperature, $36.7^{\circ} \mathrm{C}$ (normal range, $36-37^{\circ} \mathrm{C}$; respiratory rate, 18 breaths/min (normal range, $16-20$ breaths $/ \mathrm{min}$ ); blood pressure, 129/83 mmHg (normal range, 120/80 mmHg); and heart rate, 76 beats/min (normal range $60-100$ beats/min), with a regular rhythm and no pathological murmurs]. Serum calcium (Ca) on the date of admission was $3.4 \mathrm{mmol} / \mathrm{l}$ (normal range, 2.0-2.6 $\mathrm{mmol} / \mathrm{l}$ ). Alkaline phosphatase (AKP) was increased to $722 \mathrm{U} / 1$ (normal range, 15-112 U/1), and the levels of PTH in plasma were 2,630 pg/ml (normal range, $15-65 \mathrm{pg}$ / $\mathrm{ml}$ ), as measured by $\operatorname{VITROS}^{\circledR}$ ECiQ Immunodiagnostic System (Ortho Clinical Diagnostics; Johnson \& Johnson, New Brunswick, NJ, USA). However, other laboratory parameters were normal.

Neck ultrasound (CX50 CompactXtreme; Philips Healthcare, DA Best, The Netherlands) was negative for any significantly abnormal thyroid nodules or parathyroid adenoma and Tc99m methoxyisobutylisonitrile (Tc99m-MIBI) scintigraphy scanning of the parathyroid confirmed the findings of the neck ultrasound. Computed tomography (CT) of the chest, which was performed with an IQon Spectral CT (Philips Healthcare), revealed a $3.5 \times 3.0 \mathrm{~cm}$ mass originating in the anterior mediastinum, and Tc99m-MIBI scanning demonstrated increased uptake of Tc99m-MIBI on the mass, which indicated an ectopic mediastinal parathyroid adenoma (PTA; Fig. 1). Enhanced CT scanning produced a low-density image of the costal bone, right humerus and partial vertebral body. However, no widespread bone metastasis was observed.

Subsequently, the patient received intravenous hydration (10-12 1/day of $0.9 \%$ normal saline) and combined treatment with calcitonin (100 IU, i.h, q.d.; Novartis, Basel, Switzerland), alendronate sodium tablets (10 mg, p.o., q.d.; Instituto Gentili S.p.A., Milan, Italy), pamidronate disodium (30 mg, i.v. drop infusion, q.d.; Shenzhen Haiwang Pharmaceutical Co. Ltd., Guangzhou, China) and furosemide (20 mg, i.v. injection, q.d.; Huanggang Yinhe Aarti Pharmaceutical Co.,Ltd., Huangzhou, China). The day prior to surgery, the patient's serum Ca reduced to $2.42 \mathrm{mmol} / \mathrm{l}$. Therefore, all treatment that aimed to reduce serum Ca was halted. The patient underwent transthorascopic surgery to remove the giant mass in the anterior mediastinum. Surgical exploration revealed a large mass $(5 \times 3 \times 3 \mathrm{~cm}$ in size $)$ that adhered to the superior vena cava and azygos vein. Histopathological examination revealed a parathyroid carcinoma. Hematoxylin (C0107; Biyuntian Biotech Co., Ltd.) and eosin (C0109; Biyuntian Biotech Co., Ltd.) staining and immunohistochemistry (IHC) results are presented in Fig. 2, and were as follows: Cytokeratin ${ }^{+}$(mouse monoclonal antibody; dilution 1:600; ab668; Abcam, Cambridge, UK), synaptophysin $^{+}$(rabbit monoclonal antibody; dilution 1:600; ab32127; Abcam), chromogranin ${ }^{+}$(rabbit polyclonal antibody; dilution 1:400; ab15160; Abcam), human melanoma black-45 (mouse monoclonal antibody; dilution 1:50; ab787; Abcam), Melan-A (rabbit monoclonal antibody; dilution 1:200; ab51061; Abcam) and epithelial membrane antigen (rabbit monoclonal antibody; dilution 1:100; ab136615; Abcam). PTH (rabbit polyclonal antibody; dilution 1:100; ab40630; Abcam) and PTHrP (mouse monoclonal antibody; dilution 1:200; ab79539; Abcam) were additionally identified by IHC in the tumor (Fig. 2).

A total of 2 days subsequent to surgery, the patient developed perioral numbness and tingling in his extremities, and serum Ca was $1.81 \mathrm{mmol} / \mathrm{l}$. Subsequently, the patient was administered calcium carbonate and vitamin D3 tablets (600 mg, p.o., q.d.; Pfizer Inc., New York City, NY, USA), calcitriol soft capsules (0.25 $\mu$ g, p.o., q.d..; Fritz Hoffmann-La Roche AG, Basel, Switzerland) and calcium gluconate (1 g, i.v. injection, t.i.d.; Jinling Pharmaceutical Co., Ltd., Nanjing, China). With these drugs, the symptoms of numbness and tingling were resolved, and 2 months later, the patient's PTH was $65.8 \mathrm{pg} / \mathrm{ml}$ and serum Ca was $1.96 \mathrm{mmol} / 1$. There was no tumor recurrence at a 3 -year follow-up appointment, and serum $\mathrm{Ca}$ and $\mathrm{PTH}$ levels remained within normal ranges. The patient is currently under follow-up.

Written informed consent was obtained from the patient for publication of the present case report and any accompanying images.

\section{Discussion}

PTCA is a rare cause of hypercalcemia, accounting for $<1 \%$ of patients with primary hyperparathyroidism (2). In contrast to PTA, the prevalence of men is equal to women in patients with PTCA (12). The mean age at diagnosis is between 45 and 59 years, which is 10 years younger than patients with PTA (6). A total of $20-25 \%$ of parathyroid glands are in ectopic sites, and the neck and mediastinum are common ectopic sites (13). Therefore, PTCA has also been deemed as a mediastinal fifth parathyroid gland. (14).

The present study described the presentation and management of a patient with PTCA. PTCA and PTA have similar clinical and biochemical symptoms, and histological features alone often cannot entirely distinguish between them, therefore it may be difficult to diagnose PTCA (15). However, patients with PTCA have severe hypercalcemia $(>15 \mathrm{mg} / \mathrm{dl}$; normal range, 8.02-10.42 mg/dl) and markedly elevated PTH (>300 pg/ml; normal range, $15-65 \mathrm{pg} / \mathrm{ml}$ ) (2). In addition, PTCA is larger than PTA (median size, $3.3 \mathrm{~cm}$ vs. $1.5 \mathrm{~cm}$ ) (16). Furthermore, the mean levels of AKP in PTCA are typically elevated by three-fold or more (17). According to these parameters, the present patient could be diagnosed as PTCA prior to surgery. The rapid postoperative normalization of PTH levels and prolonged hypocalcemia of the present patient was most likely due to 'hungry bone syndrome', which is characterized by prolonged ( $>4$ days following parathyroidectomy) hypocalcemia (serum calcium levels, $<8.4 \mathrm{mg} / \mathrm{dl}$ ) or severe primary hyperparathyroidism as a result of extensive remineralization of bone tissue (18). In consequence, the patient received medical treatment of calcium gluconate, vitamin D3 and calcitriol. Increased PTH levels following postoperative normalization may reveal tumor recurrence. Therefore, the PTH level should be monitored during patient follow-up.

PTHrP caused hypercalcemia is widely observed in various malignancies, including lung cancer (19), breast carcinoma (20), hematological malignancies (21) and squamous cell carcinomas of the head and neck. PTHrP protein has been observed in normal parathyroid, adenomas and hyperplasia (22). However, PTHrP has not yet been reported in PTCA 
to the best of our knowledge. The present study is the first report that the tumor tissue of PTCA is able to express PTHrP, which contributes to the pathogenesis of hypercalcemia in PTCA with hypersecretion of PTH. Furthermore, PTHrP may cause hypercalcemia with normal levels of PTH (23).

The majority of clinicians recommend en bloc resection at the initial surgery to offer the best chance for cure (5), but it requires accurate positioning prior to the operation and to ascertain its nature. Although markedly elevated serum $\mathrm{Ca}$, PTH, AKP and tumor size indicate the presence of tumor tissue (2), clinicians have to decide the origin of the ectopic mass. Ultrasound is widely applied in routine inspection of the thyroid and parathyroid (24), but it could not diagnose parathyroid malignancy. However, the sensitivity of Tc99m-MIBI scintigraphy scanning combined with ultrasound is considered as high as $95 \%$ for the diagnosis of parathyroid carcinoma (25), and this technique is able to discriminate parathyroid masses from thyroid masses and higher sensitivity to distinguish malignancy from benign tumor. Diagnosis of malignant tumor, in conjunction with detailed images of enhanced CT provide, the initial surgical approach was established. Complete surgical resection is associated with an $8 \%$ local recurrence rate and a long-term overall survival rate of $89 \%$ (mean follow-up time, 69 months) (26). Adjuvant radiation therapy following surgical resection is an area of ongoing investigation due to the common local recurrence (12).

PTCA is an extremely rare disease, but it should be considered in patients with severe hypercalcemia ( $>15 \mathrm{mg} / \mathrm{dl})$, markedly elevated PTH ( $>300 \mathrm{pg} / \mathrm{ml}$ ), large tumor size (median size, $3.3 \mathrm{~cm}$ ), AKP levels elevated by threefold or greater and various symptoms of hyperparathyroidism. Tc99m-MIBI scintigraphy scanning combined with ultrasound may assist with the identification of tumor origin and nature in an ectopic mass.

In summary, the present case reported a patient with ectopic mediastinal parathyroid carcinoma cosecreting PTHrP and PTH, which contributed to hypercalcemia. It will be necessary to continue monitoring the plasma levels of PTHrP and PTH in the present patient and to perform further analyses on the different roles of PTHrP and PTH in PTCA. Currently, surgery remains the sole curative treatment for PTCA, although close clinical and radiological follow-up is advocated, due to the high risk of relapse.

\section{References}

1. Dudney WC, Bodenner D and Stack BC Jr: Parathyroid carcinoma. Otolaryngol Clin North Am 43: 441-453, 2010.

2. Fortson JK, Su R, Patel VG and Lawrence GE: Parathyroid carcinoma presenting with pathologic fracture: Case report and review of the literature. Head Neck 37: E139-E141, 2015.

3. Kettle AG and O'Doherty MJ: Parathyroid imaging: How good is it and how should it be done? Semin Nucl Med 36: 206-211, 2006

4. Damadi A, Harkema J, Kareti R and Saxe A: Use of pre-operative ${ }^{99 \mathrm{~m}} \mathrm{Tc}$-sestamibi scintigraphy and intraoperative parathyroid hormone monitoring to eliminate neck exploration in mediastinal parathyroid adenocarcinoma. J Surg Educ 64: 108-112, 2007.
5. Tseng CW, Lin SZ, Sun CH, Chen CC, Yang AH, Chang FY, Lin HC and Lee SD: Ectopic mediastinal parathyroid carcinoma presenting as acute pancreatitis. J Chin Med Assoc 76: 108-111, 2013.

6. Al-Kurd A, Mekel M and Mazeh H: Parathyroid carcinoma. Surg Oncol 23: 107-114, 2014.

7. Khan MW, Worcester EM, Straus FH II, Khan S, Staszak V and Kaplan EL: Parathyroid carcinoma in secondary and tertiary hyperparathyroidism. J Am Coll Surg 199: 312-319, 2004.

8. Harari A, Waring A, Fernandez-Ranvier G, Hwang J, Suh I, Mitmaker E, Shen W, Gosnell J, Duh QY and Clark O: Parathyroid carcinoma: A 43-year outcome and survival analysis. J Clin Endocrinol Metab 96: 3679-3686, 2011.

9. Shane E: Clinical review 122: Parathyroid carcinoma. J Clin Endocrinol Metab 86: 485-493, 2001.

10. McCauley LK and Martin TJ: Twenty-five years of PTHrP progress: From cancer hormone to multifunctional cytokine. J Bone Miner Res 27: 1231-1239, 2012.

11. Henderson JE, Shustik C, Kremer R, Rabbani SA, Hendy GN and Goltzman D: Circulating concentrations of parathyroid hormonelike peptide in malignancy and in hyperparathyroidism. J Bone Miner Res 5: 105-113, 1990.

12. Wei CH and Harari A: Parathyroid carcinoma: Update and guidelines for management. Curr Treat Options Oncol 13: 11-23, 2012.

13. Lumachi F, Zucchetta P, Varotto S, Polistina F, Favia G and D'Amico D: Noninvasive localization procedures in ectopic hyperfunctioning parathyroid tumors. Endocr Relat Cancer 6: 123-125, 1999.

14. Wynne AG, van Heerden J, Carney JA and Fitzpatrick LA: Parathyroid carcinoma: Clinical and pathologic features in 43 patients. Medicine (Baltimore) 71: 197-205, 1992

15. Minisola S, Cipriani C, Diacinti D, Tartaglia F, Scillitani A, Pepe $\mathbf{J}$ and Scott-Coombes D: Imaging of the parathyroid glands in primary hyperparathyroidism. Eur J Endocrinol 174: D1-D8, 2016.

16. Mittendorf EA and McHenry CR: Parathyroid carcinoma. J Surg Oncol 89: 136-142, 2005.

17. Hofbauer LC, Spitzweg C, Arnholdt H, Landgraf R and Heufelder AE: Mediastinal parathyroid tumor: Giant adenoma or carcinoma? Endocr Pathol 8: 161-166, 1997.

18. Ebina K, Miyoshi Y, Izumi S, Hashimoto J, Naka N, Tsukamoto Y, Kashii M, Kaito T and Yoshikawa H: A case of adolescent giant parathyroid adenoma presenting multiple osteolytic fractures and postoperative hungry bone syndrome. Clin Case Rep 3: 835-840, 2015.

19. Okagawa T and Hiramatsu Y: Parathyroid hormone-related peptide (PTHrP) producing lung cancer presenting hypercalcemia; report of a case. Kyobu Geka 68: 237-239, 2015 (In Japanese).

20. Boras-Granic K and Wysolmerski JJ: PTHrP and breast cancer: More than hypercalcemia and bone metastases. Breast Cancer Res 14: 307, 2012.

21. Clines GA and Guise TA: Hypercalcaemia of malignancy and basic research on mechanisms responsible for osteolytic and osteoblastic metastasis to bone. Endocr Relat Cancer 12: 549-583, 2005.

22. Kitazawa R, Kitazawa S, Maeda S and Kobayashi A: Expression of parathyroid hormone-related protein $(\mathrm{PTHrP})$ in parathyroid tissue under normal and pathological conditions. Histol Histopathol 17: 179-184, 2002.

23. Gurrado A, Marzullo A, Lissidini G, Lippolis A, Rubini D, Lastilla $G$ and Testini M: Substernal oxyphil parathyroid adenoma producing PTHrP with hypercalcemia and normal PTH level. World J Surg Oncol 6: 24, 2008.

24. Kunstman JW, Kirsch JD, Mahajan A and Udelsman R: Clinical review: Parathyroid localization and implications for clinical management. J Clin Endocrinol Metab 98: 902-912, 2013.

25. Gawrychowski J, Gabriel A, Kluczewska E, Buła G and Lackowska B: Mediastinal parathyroid carcinoma: A case report. Endokrynol Pol 63: 143-146, 2012.

26. Koea JB and Shaw JH: Parathyroid cancer: Biology and management. Surg Oncol 8: 155-165, 1999. 